\title{
$X$-band rf driven free electron laser driver with optics linearization
}

\author{
Yipeng Sun (孙一鹏), ${ }^{2,}{ }^{*}$ Paul Emma, ${ }^{1}$ Tor Raubenheimer, ${ }^{1}$ and Juhao $\mathrm{Wu}^{1}$ \\ ${ }^{1}$ SLAC National Accelerator Laboratory, Menlo Park, California 94025, USA \\ ${ }^{2}$ Argonne National Laboratory, Argonne, Illinois 60439, USA
}

(Received 25 March 2014; published 13 November 2014)

\begin{abstract}
In this paper, a compact hard X-ray free electron lasers (FEL) design is proposed with all X-band rf acceleration and two stage bunch compression. It eliminates the need of a harmonic rf linearization section by employing optics linearization in its first stage bunch compression. Quadrupoles and sextupoles are employed in a bunch compressor one (BC1) design, in such a way that second order longitudinal dispersion of $\mathrm{BC} 1$ cancels the second order energy correlation in the electron beam. Start-to-end 6-D simulations are performed with all the collective effects included. Emittance growth in the horizontal plane due to coherent synchrotron radiation is investigated and minimized, to be on a similar level with the successfully operating Linac coherent light source (LCLS). At a FEL radiation wavelength of $0.15 \mathrm{~nm}$, a saturation length of 40 meters can be achieved by employing an undulator with a period of $1.5 \mathrm{~cm}$. Without tapering, a FEL radiation power above $10 \mathrm{GW}$ is achieved with a photon pulse length of $50 \mathrm{fs}$, which is LCLS-like performance. The overall length of the accelerator plus undulator is around 250 meters which is much shorter than the LCLS length of 1230 meters. That makes it possible to build hard X-ray FEL in a laboratory with limited size.
\end{abstract}

DOI: 10.1103/PhysRevSTAB.17.110703

PACS numbers: 29.27.Bd, 29.20.db

\section{OVERVIEW}

Free electron lasers (FEL) were proposed by J. Madey and demonstrated for a first time at Stanford University in 1970s [1,2], using a low energy electron beam and a wiggler magnet. In a periodic wiggler magnet (or an undulator), an intense relativistic electron bunch interacts with an electromagnetic wave coherently and the radiation power is amplified exponentially. With a fixed undulator setting, the FEL radiation wavelength is tunable from the far-infrared to hard X-rays, by tuning the electron beam energy. The simplest arrangement of $\mathrm{X}$-ray lasing is to produce coherently radiated $\mathrm{X}$-rays in a long undulator by amplifying the initially noisy radiation power. This process is called self-amplified spontaneous emission (SASE) $[3,4]$. There are also other operating modes which require external seeding by laser or other technique [5].

Due to its promise in supporting wide range of research experiments, there are several other hard or soft X-ray free electron lasers being proposed in the world $[6,7,8]$, based on L-band, S-band, or C-band main rf acceleration technologies. Among them, FLASH has demonstrated the SASE FEL mechanism in principle, by successfully operating the world's first X-ray free-electron laser [6]. LCLS can work with both soft and hard X-ray modes, provide a saturated

\footnotetext{
"yisun@aps.anl.gov
}

Published by the American Physical Society under the terms of the Creative Commons Attribution 3.0 License. Further distribution of this work must maintain attribution to the author(s) and the published article's title, journal citation, and DOI.
FEL radiation in a wavelength range of 2.2 to $0.12 \mathrm{~nm}$, by tuning the electron beam energy from 3.5 to $15 \mathrm{GeV}$.

The FEL coherence condition of the electron beam in the undulator requires a high charge density, a small transverse emittance and small energy spread. Thus the electron bunch has to be compressed largely in its length during the acceleration process. Generally in the first stage of the bunch compression process where the nonlinear impacts are stronger due to an initially longer bunch length, a harmonic rf section is required to compensate the high order main rf curvature and linearize the longitudinal phase space $[9,10]$. In LCLS a fourth harmonic rf with a frequency of $11.4 \mathrm{GHz}$ is installed before bunch compressor one, to mainly minimize the second order chirp from the main $\mathrm{rf}$ which has a frequency of $2.8 \mathrm{GHz}$ [9]. In the formula below the required relation between harmonic if voltage, frequency and phase, and the main rf ones is illustrated. In the following stages of bunch compression, as the bunch length is already relatively short compared with the main rf wavelength, the nonlinear curvature from sinusoidal rf wave has a much smaller impact and then no harmonic rf linearization is necessary [11].

$V_{h} \cos \phi_{h}\left(E_{f 0} \cdot k_{h}^{2}+k^{2} \cdot e \cdot V_{0} \cos \phi\right)=-E_{f 0} k^{2} \cdot V_{0} \cos \phi$

where $V_{h}$ denotes the rf voltage of harmonic rf, $\phi_{h}$ harmonic $\mathrm{rf}$ phase, $E_{f 0}$ central energy after main $\mathrm{rf}$ acceleration, $V_{0}$ main rf voltage, $\phi_{0}$ main $\mathrm{rf}$ phase, $k_{h}=$ $\frac{2 \pi f_{h}}{c}$ the harmonic rf wave number, $k=\frac{2 \pi f}{c}$ the main rf wave 


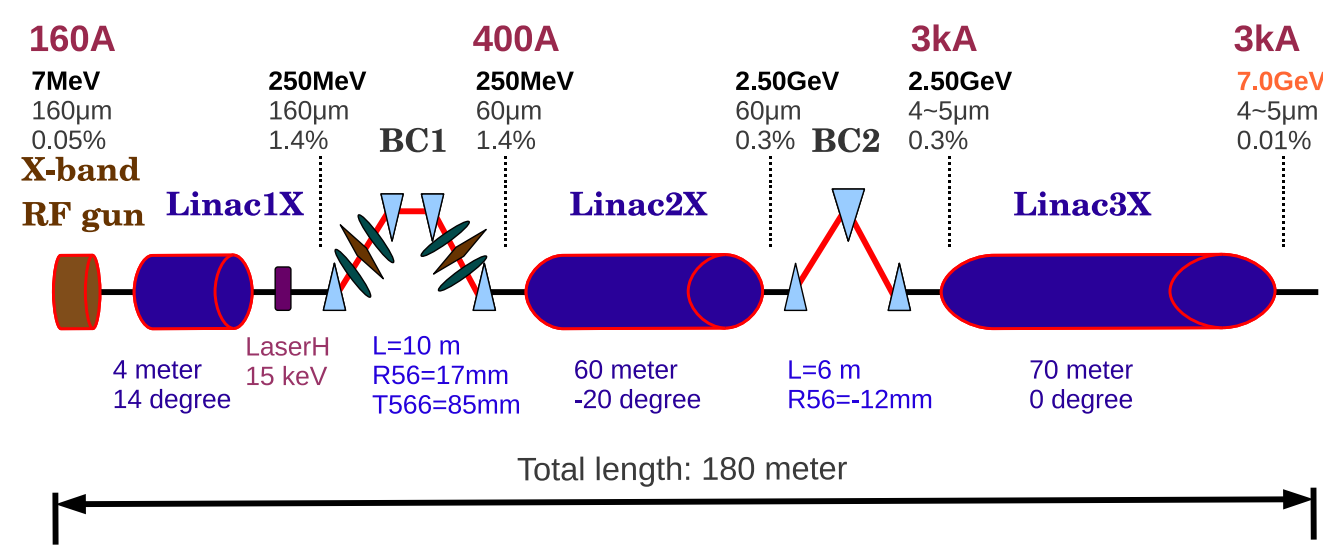

FIG. 1. Sketch of an all X-band based hard X-ray FEL accelerator design (BC1 is sketched as a chicane shape here).

number. The main argument against a higher harmonic system to an $\mathrm{X}$-band linac is that it is not available.

Chicane and wiggler based bunch compressors were proposed and studied thoroughly in the 1990s, mainly for several linear collider projects $[12,13,14]$. In this paper, an alternative way to do linearized bunch compression in a first stage is proposed. The key point is to eliminate the harmonic rf section, and to apply an optics linearization instead, with a specially designed bunch compressor which includes quadrupole and sextupole magnets. Similar schemes have been proposed and studied preliminarily before, either in an analytical manner $[14,15,16]$, by numerical simulations $[14,16,17,18]$, or in experimental measurements [16]. The second order longitudinal dispersion $T_{566}$ of this bunch compressor is precisely controlled and used to compensate the second order curvature from the main $\mathrm{rf}$ acceleration. Third order longitudinal dispersion $U_{5666}$ can also be tuned to cancel the third order rf curvature, however, in most cases this is not necessary as its impact is relatively small. This approach is especially interesting for X-band FEL drivers, as it is harder to find and operate harmonic rf, i.e., above $30 \mathrm{GHz}$.

In the following sections, basic formulas of longitudinal motion and bunch compression are derived analytically. A hard X-ray FEL design which is based on all X-band rf system is presented. Optics design of BC1 is performed analytically using a matrix approach, and with the accelerator code MAD8 [19]. A start-to-end 6-D simulation is performed in ELEGANT [20] with all the collective effects included. That is followed by FEL simulation with photon energy of $8 \mathrm{keV}$ in GENESIS [21]. The accelerator of this FEL driver is sketched in Fig. 1.

\section{MAGNETIC BUNCH COMPRESSION}

Magnetic bunch compression is to establish an energy correlation along the bunch longitudinal axis, then let the beam pass by a dispersive region in which particles with different energy have different path length. This dispersive region can either be a normal four dipole chicane, or a bunch compressor with multipole magnets as introduced in the following sections.

\section{A. rf chirp}

The energy correlation (chirp) is established by rf acceleration on an off crest phase. For any particle in an electron bunch, its relative energy offset after passing by this rf section can be expressed as a function of its longitudinal coordinate $z$, as shown in Eq. (2).

$$
\begin{aligned}
\delta(z) & =\delta_{i} \frac{E_{i 0}}{E_{f 0}}+\frac{e V_{0} \cos \left(\phi+k z_{i}\right)}{E_{f 0}} \\
& =a \cdot \delta_{i}+\frac{e V_{0} \cos \left(\phi+k z_{i}\right)}{E_{f 0}}
\end{aligned}
$$

where $\delta_{i}$ denotes the initial uncorrelated energy offset, $E_{i 0}$ central energy before rf acceleration, $E_{f 0}$ central energy after $\mathrm{rf}$ acceleration, $e$ electron charge, $V_{0}$ the $\mathrm{rf}$ voltage, $\phi$ the rf phase, $k=\frac{2 \pi}{\lambda}$ the $\mathrm{rf}$ wave number, $\lambda$ the rf wavelength, $z_{i}$ particle's longitudinal coordinate relative to the bunch center and $a=\frac{E_{i 0}}{E_{f 0}}$ energy ratio or damping factor.

One then could easily derive an energy chirp up to third order as expressed in the following formulas, assuming that an initial beam centroid energy is very small with respect to the final beam energy $\left(\Delta E \approx E_{f 0}\right)$.

$$
\begin{aligned}
& h_{1}=-\frac{k e V_{0} \sin \phi}{E_{f 0}}=-k \frac{\Delta E}{E_{f 0}} \tan \phi \approx-k \tan \phi, \\
& h_{2}=-\frac{k^{2} e V_{0} \cos \phi}{2 E_{f 0}}=-\frac{k^{2} \Delta E}{2 E_{f 0}} \approx-\frac{k^{2}}{2}, \\
& h_{3}=\frac{k^{3} e V_{0} \sin \phi}{6 E_{f 0}}=\frac{k^{3}}{6} \frac{\Delta E}{E_{f 0}} \tan \phi \approx \frac{k^{3}}{6} \tan \phi,
\end{aligned}
$$

where $e$ denotes the electron charge, $\Delta E$ the energy gain through rf acceleration. One observes that in general the 
chirp is proportional to rf frequency, also it is a function of rf phase.

\section{B. Dispersive region}

After passing by a dispersive region, the longitudinal coordinate $z$ of any electron with respect to the bunch center can be expressed as a function of its relative energy offset $\delta$, as shown below.

$$
\begin{aligned}
z_{f}(\delta) & =z_{i}+R_{56} \delta+T_{566} \delta^{2}+U_{5666} \delta^{3}+\cdots \\
& =z_{i}+b_{1} \delta+b_{2} \delta^{2}+b_{3} \delta^{3}+\cdots
\end{aligned}
$$

where $z_{i}$ denotes an initial longitudinal coordinate relative to the bunch center, $b_{1}=R_{56}$ a first order longitudinal dispersion, $b_{2}=T_{566}$ a second order longitudinal dispersion, $\delta$ relative energy offset, and $b_{3}=U_{5666}$ a third order longitudinal dispersion.
Insert Eq. (2) into Eq. (6) (neglect the relatively small initial uncorrelated energy offset in the higher order terms), the final longitudinal coordinate of any particle is expressed in Eq. (7).

$$
\begin{aligned}
z_{f}= & z_{i}+b_{1}\left(a \cdot \delta_{i}+h_{1} z_{i}+h_{2} z_{i}^{2}+h_{3} z_{i}^{3}\right) \\
& +b_{2}\left(h_{1} z_{i}+h_{2} z_{i}^{2}+h_{3} z_{i}^{3}\right)^{2} \\
& +b_{3}\left(h_{1} z_{i}+h_{2} z_{i}^{2}+h_{3} z_{i}^{3}\right)^{3}+\cdots
\end{aligned}
$$

\section{Bunch length after compression}

Assuming that the electron bunch preserves a Gaussian distribution after passing by a dispersive region, in Eq. (7), keep the terms up to second order (neglect the initial uncorrelated energy offset $\delta_{i}$ in higher order terms) one finds that the square of any electron's final longitudinal coordinate $z_{f}^{2}(z, \delta)$ equals

$$
\begin{aligned}
z_{f}^{2}\left(z, \delta_{i}\right)= & a^{2} R_{56}^{2} \delta_{i}^{2}+2 R_{56} \cdot a \cdot \delta_{i}\left(1+h_{1} R_{56}\right) \cdot z+\left[\left(1+h_{1} R_{56}\right)^{2}+2 R_{56} \cdot a \cdot \delta_{i}\left(h_{2} R_{56}+h_{1}^{2} \cdot T_{566}\right)\right] \cdot z^{2}+(\cdots) \cdot z^{3} \\
& +\left[\left(h_{1}^{2} \cdot T_{566}+h_{2} \cdot R_{56}\right)^{2}+4 h_{1} h_{2} T_{566}\left(1+h_{1} R_{56}\right)+2 h_{2}^{2} R_{56} T_{566} \cdot a \cdot \delta_{i}\right] \cdot z^{4}+(\cdots) \cdot z^{5}+(\cdots) \cdot z^{6} \\
& +(\cdots) \cdot z^{7}+(\cdots) \cdot z^{8}
\end{aligned}
$$

where $\delta_{i}$ denotes the initial uncorrelated energy offset, $z_{f}(z, \delta)$ the final longitudinal coordinate, and $z$ the initial longitudinal coordinate.

In Eq. (7) keeping the terms up to third order, again one finds that the square of any electron's final longitudinal coordinate $z_{f}^{2}(z, \delta)$ equals

$$
\begin{aligned}
z_{f}^{2}\left(z, \delta_{i}\right)= & a^{2} R_{56}^{2} \delta_{i}^{2}+2 R_{56} \cdot a \cdot \delta_{i}\left(1+h_{1} R_{56}\right) \cdot z+\left[\left(1+h_{1} R_{56}\right)^{2}+2 R_{56} \cdot a \cdot \delta_{i}\left(h_{2} R_{56}+h_{1}^{2} \cdot T_{566}\right)\right] \cdot z^{2}+(\cdots) \cdot z^{3} \\
& +\left[\left(h_{1}^{2} \cdot T_{566}+h_{2} \cdot R_{56}\right)^{2}+4 h_{1} h_{2} T_{566}\left(1+h_{1} R_{56}\right)+2 h_{2}^{2} R_{56} T_{566} \cdot a \cdot \delta_{i}\right. \\
& +\left[\left(4 h_{1} R_{56} T_{566} \cdot a \cdot \delta_{i}+2 R_{56}\left(1+h_{1} R_{56}\right)\right] \cdot h_{3}+6 h_{1}^{2} h_{2} R_{56} U_{5666} \cdot a \cdot \delta_{i}+2 h_{1}^{3} U_{5666}\left(1+h_{1} R_{56}\right)\right] \cdot z^{4} \\
& +(\cdots) \cdot z^{5}+\left[(\cdots) \cdot a \cdot \delta_{i}+(\cdots) \cdot\left(1+h_{1} R_{56}\right)+\left(h_{3} R_{56}+2 h_{1} h_{2} T_{566}+h_{1}^{3} U_{5666}\right)^{2}\right] \cdot z^{6} \\
& +(\cdots) \cdot z^{7}+(\cdots) \cdot z^{8}+\cdots+(\cdots) \cdot z^{18}
\end{aligned}
$$

where $\delta_{i}$ denotes the initial uncorrelated energy offset, $z_{f}(z, \delta)$ the final longitudinal coordinate, and $z$ the initial longitudinal coordinate.

\section{Linearization by choosing proper $R_{56}, T_{566}$ and $U_{5666}$}

Given the condition that the first order coefficient $a_{1}$ is zeroed by choosing a proper rf phase and linear dispersion $R_{56}, a_{1}=1+b_{1} \cdot h_{1}=0$, one finds

$$
R_{56}=b_{1}=-\frac{1}{h_{1}}=\frac{E_{f 0}}{k \Delta E \tan \phi}
$$

where $\Delta E$ denotes the energy gain through rf acceleration, $E_{f 0}$ final beam energy, $k$ rf wave number and $\phi$ rf phase.
With $a_{1}=0$ and a negligible uncorrelated initial energy spread $\sigma_{\delta i}$, the contribution to $z^{4}$ from all the cross terms is zero, as illustrated in Eqs. (8) and (9). Under these conditions, by letting the coefficient of the 2 nd order term $a_{2}=0$, one finds the required second order longitudinal dispersion $T_{566}$ as a function of linear dispersion $R_{56}$, as shown below.

$$
T_{566}=b_{2}=-\frac{h_{2}}{h_{1}^{2}} \cdot R_{56}=\frac{R_{56}}{2} \cdot \frac{E_{f 0}}{\Delta E} \cdot \frac{1}{\tan \phi^{2}}
$$

where $\Delta E$ denotes the energy gain through rf acceleration, $E_{f 0}$ final beam energy, and $\phi$ rf phase.

Similarly, with $a_{1}=0, a_{2}=0$, the contribution to $z^{6}$ from all the cross terms is zero too, as illustrated in Eq. (9). 
By letting $a_{3}=0$ one finds the required third order longitudinal dispersion $U_{5666}$ as shown below.

$$
U_{5666}=b_{3}=R_{56} \cdot \frac{E_{f 0}^{2}}{\Delta E^{2}} \cdot\left(\frac{1}{6 \tan ^{2} \phi}+\frac{1}{2 \tan ^{4} \phi}\right)
$$

where $\Delta E$ denotes the energy gain through rf acceleration, $E_{f 0}$ final beam energy and $\phi$ rf phase.

One needs to note that the above derived dispersion formulas are only valid for full compression $\left(a_{1}=1+b_{1} \cdot h_{1}=0\right)$. In practice, usually undercompression or overcompression is adopted $\left(a_{1}=1+b_{1} \cdot h_{1} \neq 0\right)$ in each compression stage, and the required bunch compressor dispersions $\left(R_{56}, T_{566}\right.$ and $\left.U_{5666}\right)$ need to be calculated according to the real condition. This will be further discussed in the bunch compressor optics design section below.

\section{E. RMS energy spread after compression}

Keep third order terms and apply an approximation, one then finds that the square of energy offset of any electron can be expressed as in the following formula.

$$
\begin{aligned}
\delta_{f}^{2}= & \left(a \cdot \delta_{i}\right)^{2}+h_{1}^{2} z^{2}+2 h_{1} h_{2} z^{3}+\left(2 h_{1} h_{3}+h_{2}^{2}\right) z^{4} \\
& +2 h_{2} h_{3} z^{5}+h_{3}^{2} z^{6} .
\end{aligned}
$$

The final RMS energy spread of the electron bunch is calculated by integration and listed in the following formula.

$$
\begin{aligned}
\sigma_{\delta}^{2}= & a^{2} \sigma_{\delta i}^{2}+h_{1}^{2} \cdot \sigma_{z, i}{ }^{2}+3 \cdot\left(2 h_{1} h_{3}+h_{2}^{2}\right) \cdot \sigma_{z, i}{ }^{4} \\
& +15 \cdot h_{3}^{2} \cdot \sigma_{z, i}{ }^{6} .
\end{aligned}
$$

One observes that a final RMS energy spread is always larger if higher order terms are included.

\section{AN ALL X-BAND HARD X-RAY FEL}

The accelerator starts with an X-band photoinjector, which promises to generate an electron bunch with low transverse emittance and short bunch length. A bunch charge of $250 \mathrm{pC}$ is adopted which RMS length can be as short as $160 \mu \mathrm{m}$, and its normalized transverse emittance is under $0.5 \mu \mathrm{mrad}$ [22]. The other systems will be discussed below.

\section{A. Bunch compressor one with optics linearization}

The first stage bunch compression is the most important part in this all X-band hard X-ray FEL design. After the electron bunch length has been compressed in a first stage bunch compression, the nonlinear rf curvature from the following linac acceleration tends to be small and can be neglected [11]. As discussed in Sec. II, one needs specific $R_{56}$ and $T_{566}$ to do optics linearization. In the following part, it is discussed how to achieve that in a bunch compressor design. Starting from the equation of motion and dispersion function, one could derive a relation between longitudinal dispersion and dispersion in the bending plane (from the difference in path length between an on-momentum particle and an off-momentum particle), as shown below [15].

$$
R_{56}=\int_{0}^{s 0} \frac{R_{16}}{\rho_{0}} d s
$$

where $R_{56}$ denotes the first order longitudinal dispersion, $R_{16}$ the first order horizontal dispersion, and $\rho_{0}$ the bending curvature in a constant dipole magnet. It is observed that the first order longitudinal dispersion $R_{56}$ only depends on the horizontal dispersion $R_{16}$ in the dipole magnets and the bending radius.

One can prove that for any bunch compressor which is composed of only dipole magnets and drift spaces, its first order longitudinal dispersion $R_{56}$ is always negative. In order to freely tune first order longitudinal dispersion $R_{56}$ and linear compression ratio, one needs the assistance of quadrupole magnets. After adding the quadrupole magnets, it is still possible to find an optics solution which closes the dispersion function $R_{16}$ and angular dispersion function $R_{26}$ in the bending plane. However, only closing first order dispersion terms in the bending plane is not enough, as there will be emittance growth due to a large correlated energy spread and residual high order horizontal dispersions in the bending plane (i.e., $T_{166}$ and $T_{266}$ ) [23]. As a result, sextupole magnets have to be introduced in the bunch compressor beam line, near the quadrupole magnets, to tune the second order dispersions and make them zero at the end of the bunch compressor. Usually one can maintain a midplane mirror-symmetry when one arranges a sequence of these magnets such as dipole, quadrupole, sextupole, and drift spaces, and find an optics solution.

As discussed above, one also needs a specified second order longitudinal dispersion $T_{566}$ to do optics linearization. Similar as the first order dispersion derivation, a relation between longitudinal and transverse second order dispersion terms can be derived (again from the path length difference), as shown below [15].

$$
T_{566}=\int_{0}^{s 0}\left[\frac{T_{166}}{\rho_{0}}+\frac{1}{2} R_{26}^{2}+\frac{1}{2}\left(\frac{R_{16}}{\rho_{0}}\right)^{2}\right] d s
$$

where $R_{26}$ denotes the first order horizontal angular dispersion, $T_{566}$ the second order longitudinal dispersion and $T_{166}$ the second order horizontal dispersion. One then observes that for a midplane symmetric and finally closed second order horizontal dispersion $T_{166}$, the integral $\int_{0}^{s 0} \frac{T_{166}}{\rho_{0}} d s$ equals two times of the integral in a half bunch compressor. The second order longitudinal dispersion $T_{566}$ also depends on the other two terms, which are the first 
order horizontal dispersion $R_{16}$ and horizontal angular dispersion $R_{26}$.

Following the analytical derivations in Sec. II D above, here the required second order longitudinal dispersion $T_{566}$ to linearize the longitudinal phase space is discussed for either an undercompression or an overcompression case. In either case, one finds that $1+b_{1} \cdot h_{1} \neq 0$, which is dependent on the linear bunch compression ratio adopted. In Eq. (7), keep the second order terms, one finds that the required $T_{566}$ can be solved by letting the second order energy chirp equal zero.

$$
\begin{aligned}
& \left(h_{1}^{2} \cdot T_{566}+h_{2} \cdot R_{56}\right)^{2}+4 h_{1} h_{2} T_{566}\left(1+h_{1} R_{56}\right) \\
& \quad+2 h_{2}^{2} R_{56} T_{566} \cdot a \cdot \delta_{i}=0 .
\end{aligned}
$$

From the above equation one finds that the solution of $T_{566}$ mainly depends on the first order and second order chirp, plus the first order longitudinal dispersion $R_{56}$. Employing the given X-band $\mathrm{rf}$ and incoming beam parameters, a second order longitudinal dispersion of $T_{566}=80 \mathrm{~mm}$ is required to linearize the longitudinal phase space, for an overcompression with a compression ratio of 4 (a first order longitudinal dispersion of $R_{56}=17 \mathrm{~mm}$ ).

A matrix approach $[24,25]$ is adopted here to analytically investigate first and second order optics [23], which is composed of dipole, quadrupole, sextupole magnets, and drift spaces. Thin lens approximation is applied here for all the analytical derivations. In first order, the transport of any particle's six coordinates can be treated in a general matrix formula as shown below $\mathbf{X}=\mathbf{R}_{[6 \times 6]} \cdot \mathbf{X}_{\mathbf{0}}$, where $\mathbf{R}$ denotes the first order transport matrix of any beam line. Second order optics is treated in a similar manner. The acquired magnets parameters from analytical plus numerical manipulations and estimation, which fulfills the requirement on the transport matrix elements $R_{16}, R_{26}, R_{56}, T_{166}, T_{266}$, and $T_{566}$, are then matched again numerically in an accelerator design code MAD8 [19].

Several different configurations of $\mathrm{BC} 1$ are studied and evaluated by their beam dynamics performance. Finally, a dogleg based bunch compressor is chosen to be a baseline design, due to its simple shape and flexibility. There are four 0.2-meter-long dipole magnets in this beam line, where the outer two dipole magnet bends for 7 degree each and the inner two bends for 3 degree each. Overcompression is adopted in this first stage bunch compression. Full compression happens in the middle of the third dipole magnet, as the two inner dipole magnets dominant the longitudinal dispersion $R_{56}$. A short dipole magnet length of 0.2 meter has an advantage in achieving a short interaction time of coherent synchrotron radiation (CSR) and a fast turn over the full compression region, which better preserves transverse emittance. Another point is that large dispersion in the third dipole, plus large energy spread generate a large transverse beam size, which provides transverse suppression of CSR impacts near full compression point. In other words, the effective projected bunch length is longer in the direction of radiation.

CSR is a process that the photons radiated by the electron bunch tail catches the bunch head, and it is coherent for wavelengths greater than the bunch length. The RMS energy spread introduced by the CSR effect in a single dipole magnet could be approximated as shown below.

$$
\left(\frac{\Delta E}{E}\right)_{r m s} \approx 0.22 \frac{r_{e} N L}{\gamma R^{2 / 3} \sigma_{z}^{4 / 3}}
$$

where $r_{e}$ denotes classical electron radius, $N$ electron bunch population, $L$ length of the dipole magnet, $\gamma$ relativistic beam energy, $R$ bending radius and $\sigma_{z}$ RMS electron bunch length. One observes that CSR is relatively strong at low beam energy, such as at $\mathrm{BC} 1$.

In general, CSR effect is longitudinally correlated when it is evaluated over a very short range and time scale. Every longitudinal slice of the electron bunch has a different energy change and then has different trajectory in the bunch compressor. Although one could employ tuning quadrupole magnets in the bunch compressor and steering corrector magnets afterwards to zero the average trajectory and angle, the transverse projected emittance of the whole bunch is increased as the motion of different slice is not "similar" anymore.

Next the physical mechanism of sliced emittance growth is briefly addressed here. Assume that $s$-correlated energy change is introduced by CSR effect ( $s$ is longitudinal coordinate inside the bunch), and there is no phase space rotation (smear) in this process, one finds that the final horizontal coordinate and divergence of any electron could be expressed as shown below.

$$
\begin{aligned}
x= & x_{0}+\left(R_{16} \cdot \delta_{0}+\overline{R_{16}} \cdot \Delta \delta(s)\right)+\left(T_{166} \cdot \delta_{0}^{2}+\overline{T_{166}} \cdot \Delta \delta(s)^{2}\right) \\
& +\left(T_{161} \cdot \delta_{0}+\overline{T_{161}} \cdot \Delta \delta(s)\right) \cdot x_{0} \\
& +\left(T_{162} \cdot \delta_{0}+\overline{T_{162}} \cdot \Delta \delta(s)\right) \cdot x_{0}^{\prime}+\cdots
\end{aligned}
$$

where $R_{16}, T_{166}, T_{161}$, and $T_{162}$ denote first order and second order transfer matrix elements, $\overline{R_{16}}, \overline{T_{166}}, \overline{T_{161}}$, and $\overline{T_{162}}$ the effective coefficients associated with CSR effect, $\delta_{0}$ initial relative energy offset, $\Delta \delta(s)$ relative energy loss introduced by CSR effect and $s$ longitudinal coordinate in the electron bunch.

$$
\begin{aligned}
x^{\prime}= & x_{0}^{\prime}+\left[R_{26} \cdot \delta_{0}+\overline{R_{26}} \cdot \Delta \delta(s)\right]+\left[T_{266} \cdot \delta_{0}^{2}+\overline{T_{266}} \cdot \Delta \delta(s)^{2}\right] \\
& +\left[T_{261} \cdot \delta_{0}+\overline{T_{261}} \cdot \Delta \delta(s)\right] \cdot x_{0} \\
& +\left[T_{262} \cdot \delta_{0}+\overline{T_{262}} \cdot \Delta \delta(s)\right] \cdot x_{0}^{\prime}+\cdots
\end{aligned}
$$

where $T_{261}$ and $T_{262}$ denote second order transfer matrix elements.

One observes that if the initial uncorrelated energy offset $\delta_{0}$ is small and negligible, then the change on any electron's 

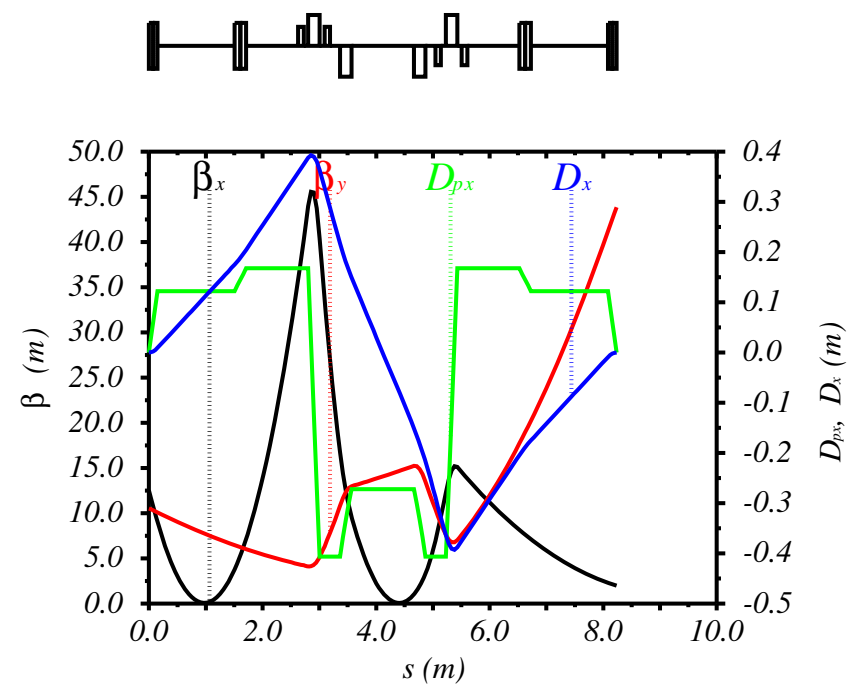

FIG. 2. First order optics of bunch compressor one. Black curve denotes horizontal beta function, red curve vertical beta function, blue curve horizontal dispersion function, and green curve horizontal angular dispersion function.

horizontal coordinate and divergence only depends on the correlated term $\Delta \delta(s)$. As $\Delta \delta(s)$ is the same for electrons in the same slice, it only changes the slice trajectory, not the emittance.

On the other hand, if there are both s-correlated energy change and phase space rotation (smear) happening at the same time, such as the overcompression in a first stage of this $\mathrm{X}$-band based FEL, one finds that $\Delta \delta(s)$ is not the same even for electrons in the same slice. One observes that for this case, even the electrons in the same slice have different overall energy loss, which means they also have different trajectory change. The longitudinally sliced horizontal emittance is increased accordingly. This effect is more severe when higher order energy chirp and dispersions are present, especially for overbunch compression.

As shown by the first order optics in Fig. 2, two focusing quadrupole magnets and two defocusing quadrupole magnets are introduced between the two inner dipole magnets, in a mirror symmetric manner. The quadrupole length is 0.2 meter, with a maximum normalized strength of $K 1=7 \mathrm{~m}^{-1}$. The relatively strong focusing quadrupole magnet which is located at a maximum dispersion position inverts the sign of angular dispersion $R_{26}$. The slope of $R_{16}$ is then slightly modified by a defocusing quadrupole magnet. At the middle point of this dogleg, the horizontal dispersion $R_{16}$ equals zero. Again these optimizations are based on CSR calculations, as the bunch length is the shortest in the middle of the third dipole magnet (full compression) and it remains relatively short to the end of the bunch compressor. A small beta function sets the electron bunch at a waist in this region, which in turn makes the CSR induced emittance growth smaller as this effect mainly change beam's divergence $\left(x^{\prime}\right)$.
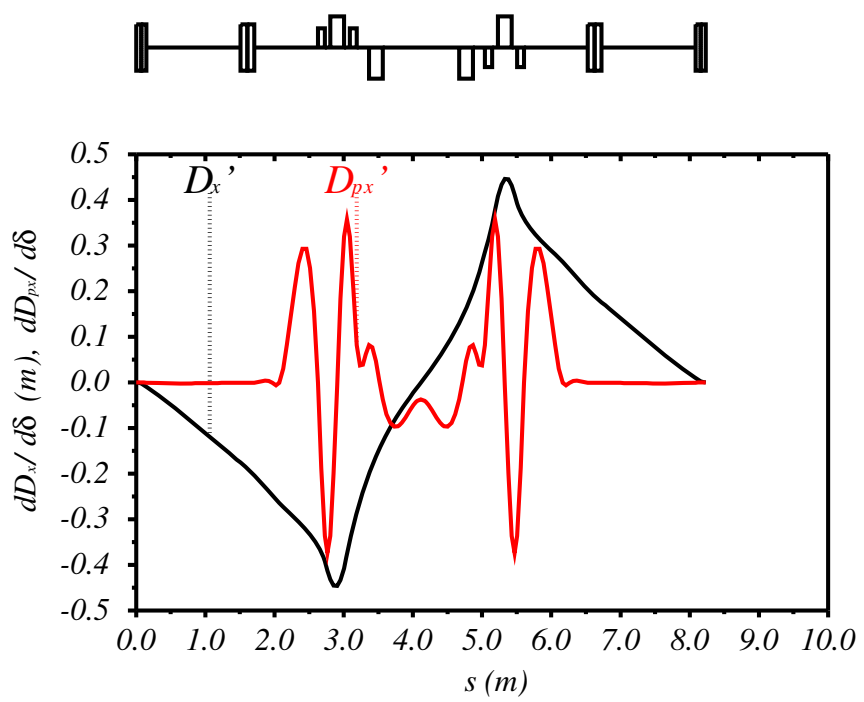

FIG. 3. Second order optics of bunch compressor one. Black curve denotes second order horizontal dispersion function and red curve second order horizontal angular dispersion function.

Second order optics of this dogleg beam line is shown in Fig. 3, where one observes that four sextupole magnets are introduced to tune the second order optics. The sextupole magnet has a length of 0.1 meter and a maximum normalized strength of $K 2=50 \mathrm{~m}^{-1}$. As mentioned they are arranged in a mirror symmetric type and all of them are focusing sextupole magnets. At the end of this dogleg beamline, the second order horizontal dispersion $T_{166}$ and angular dispersion $T_{266}$ are closed, $T_{166}=0$ and $T_{266}=0$.

To summarize, this dogleg beamline could successfully serve as a bunch compressor which provides the required longitudinal dispersions, $R_{56}=17 \mathrm{~mm}$ and $T_{566}=80 \mathrm{~mm}$. The first order and second order dispersions in the bending plane are closed at the end, which suppresses possible dispersive emittance growth associated with a large correlated energy spread of the electron bunch.

\section{B. ELEGANT 6-D simulation}

In this section start-to-end ELEGANT [20] 6-D numerical simulation results are presented. The simulation starts at a beam energy of $50 \mathrm{MeV}$. X-band photoinjector simulations show that an RMS bunch length of $160 \mu \mathrm{m}$ could be achieved along with a normalized transverse emittance of $0.5 \mu \mathrm{m} \mathrm{rad}$ at a bunch charge of $250 \mathrm{pC}$ [22]. The uncorrelated RMS energy spread is $12.5 \mathrm{keV}$. This electron bunch is transported through a first linac section (Linac1), bunch compressor one, a second linac section (Linac2), bunch compressor two and a third linac section (Linac3). The start-to-end first order optics is illustrated in Fig. 4, from entrance of Linac1 to the end of Linac3.

In Linac1 the beam is accelerated from $50 \mathrm{MeV}$ to $250 \mathrm{MeV}$, on a rf phase of positive 14 degree. A falling rf slope is adopted here as the first dogleg bunch compressor 


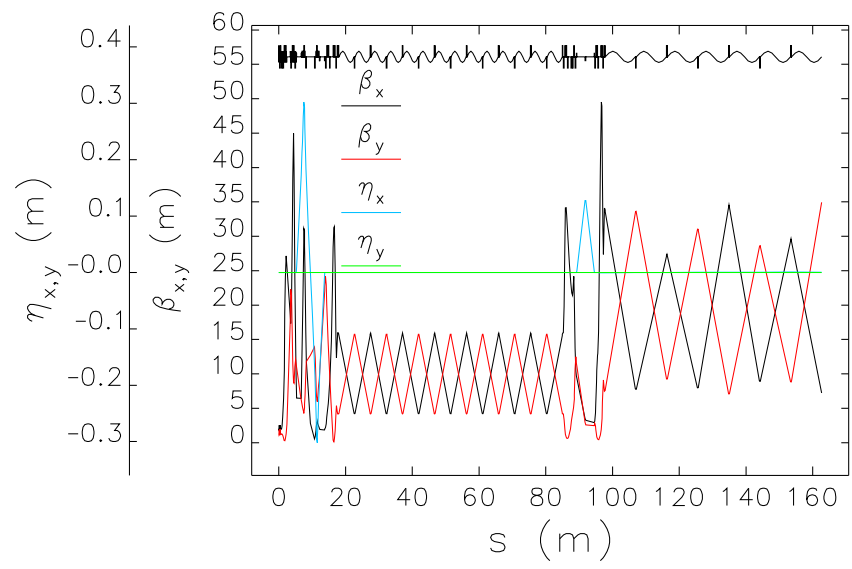

FIG. 4. Start-to-end TWISS parameters, a beam energy from $50 \mathrm{MeV}$ to $7 \mathrm{GeV}$. Black curve denotes horizontal beta function, red curve vertical beta function, blue curve horizontal dispersion function, and green curve horizontal angular dispersion function.

has a positive first order longitudinal dispersion $R_{56}$. Periodic FODO cell is employed in all three linac sections. The distance between two quadrupoles is $4.5 / 9$ meters in Linac2/Linac3 and the betatron phase advance is $72 / 72$ degree per cell. As can be observed in Fig. 4, the average beta function is 10 meters and 20 meters in Linac2 and Linac3, respectively. A laser heater is installed between Linac1 and bunch compressor one, to provide an additional uncorrelated energy spread of $15 \mathrm{keV}$ for stronger Landau damping. Longitudinal and transverse wake field [26], as well as longitudinal space charge effect are included in the simulation.

As discussed in Sec. III A, a required first order longitudinal dispersion of $R_{56}=17 \mathrm{~mm}$ and a second order longitudinal dispersion of $T_{566}=80 \mathrm{~mm}$ are achieved in the first stage dogleg bunch compressor. The second order energy correlation is minimized in the bunch compression process. The impacts from third order energy chirp and third order longitudinal dispersion on the bunch length and distribution are small and not considered here. The electron bunch is quasilinearly overcompressed in this first stage dogleg bunch compressor. The RMS bunch length is $50 \mu \mathrm{m}$ at the end of the bunch compressor one, and the RMS correlated energy spread is $1.4 \%$.

One matching section of four quadrupole magnets is employed on each side of the bunch compressor. Its main function is to match the beta functions between linac section and bunch compressors. The maximum beta function is constrained to be as small as possible in this region to reduce CSR influence. In ELEGANT simulation the trajectory and the average angle of the electron beam is manually corrected to be zero, while in real operation this could be done with steering magnets. The electron bunch is accelerated to a beam energy of $2.5 \mathrm{GeV}$ in 60 meters Linac2. The rf phase is negative 15 degrees in Linac2 which means a rising rf slope is employed. The longitudinal wake field in Linac2 generates an inverse energy chirp compared with the rf induced energy correlation. This effect helps to cancel the timing jitter (correlated among all linac rf systems) between photoinjector drive laser phase and linac rf phase. It is shown that one could adopt a longer Linac2 with lower acceleration gradient to fully suppress the timing jitter effect, even for X-band rf which is more sensitive to timing jitter [27].

The electron bunch is next injected into bunch compressor two which is a four dipole chicane. Relatively weak dipole magnets are used which length is 0.4 meters and generates a first order longitudinal dispersion of $R_{56}=-17 \mathrm{~mm}$. The bunch length is compressed 10 times in this second stage to a final RMS value of $17 \mathrm{fs}$. The horizontal beta function in both bunch compressors is optimized based on the experience of LCLS design and operation [7]. The evolvement of longitudinal phase space of the electron bunch is illustrated in Figure 5.

The electron beam brightness has to be preserved during the acceleration and compression process for a finally successful lasing in the undulator. For a perfectly aligned linear accelerator, the impact on transverse emittance from transverse wake field is small and negligible. Above an electron beam energy of $250 \mathrm{MeV}$ after bunch compressor one, the space charge effect is also weak and has little impact on the transverse emittance. The longitudinal wake field effect in the linac generates an additional energy correlation which needs to be considered when choosing the rf parameters. The transverse emittance growth is mainly from CSR impacts which happens in the bunch compression process.

The transverse emittance evolution is shown in Fig. 6. One observes that while the vertical emittance is always preserved during the acceleration and bunch compression, the horizontal emittance is increased by $10 \%$ in the core part and 30\% in the bunch head and tail. The horizontal emittance growth in the core part mainly happens in the first stage bunch compression, and in the second stage bunch compression the emittance is increased in the head and tail. The CSR induced transverse emittance growth is on a similar level of the LCLS.

After two stage bunch compression, a peak current above $3 \mathrm{kA}$ is achieved in a pulse length of $50 \mathrm{fs}$. The vertical normalized emittance is preserved at $0.5 \mu \mathrm{m} \mathrm{rad}$, and the horizontal normalized emittance is increased by $20 \%$ in average. The residual correlated energy spread is removed in Linac3, mainly by the strong X-band rf longitudinal wake field. The Linac3 runs on-crest with a total length of 70 meters. A final flat energy profile is achieved as shown in Fig. 7, as well as the current profile, longitudinally sliced transverse emittance and energy spread. This final electron beam is injected into the design undulator for hard $\mathrm{X}$-ray FEL with a radiation wavelength as short as $0.15 \mathrm{~nm}$. The detailed FEL simulation and performance is presented below. 

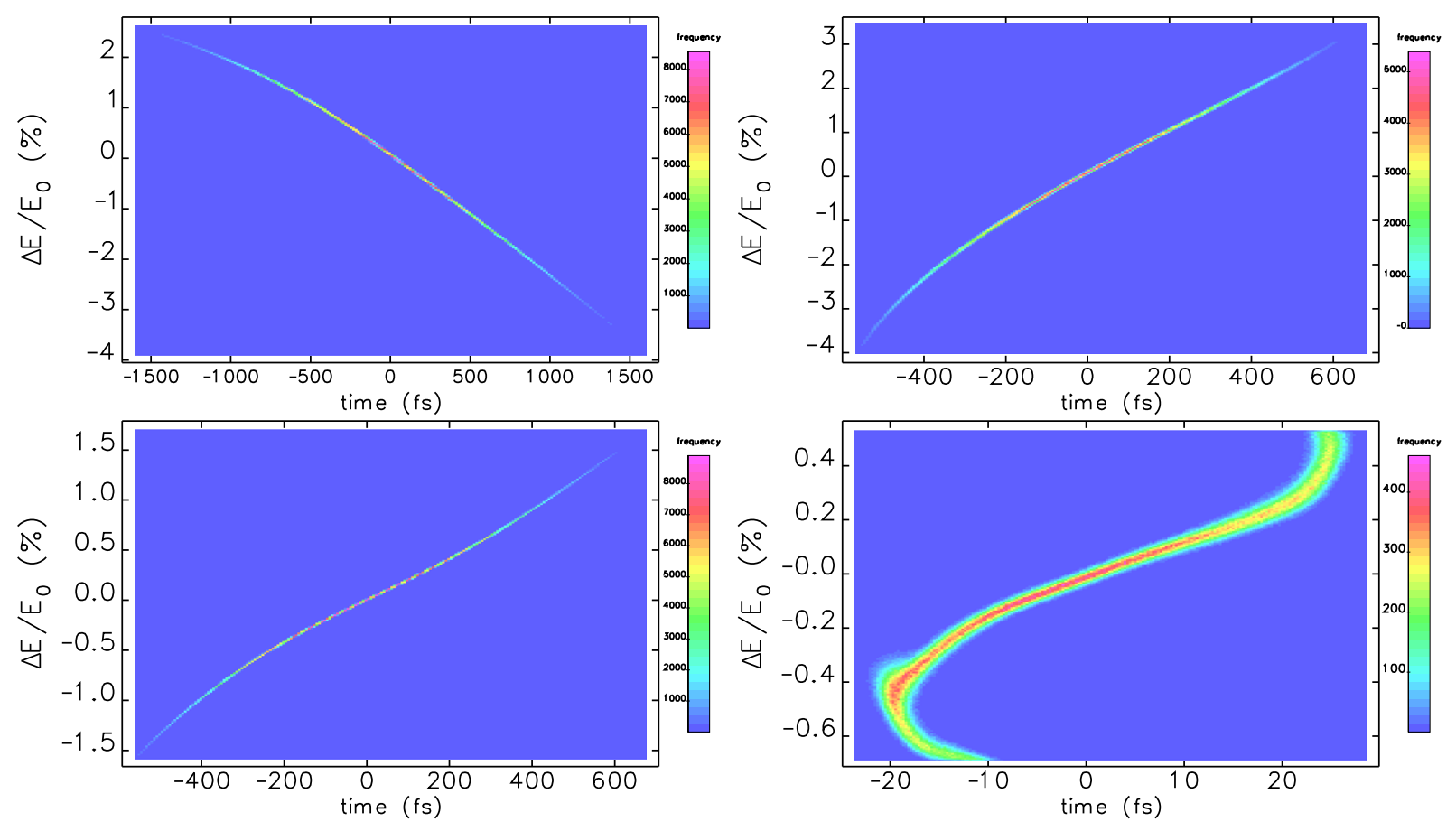

FIG. 5. Longitudinal phase space evolution. Left top: Linac1 end and BC1 entrance; right top: BC1 end; left bottom: Linac2 end and BC2 entrance; left bottom: BC2 end.
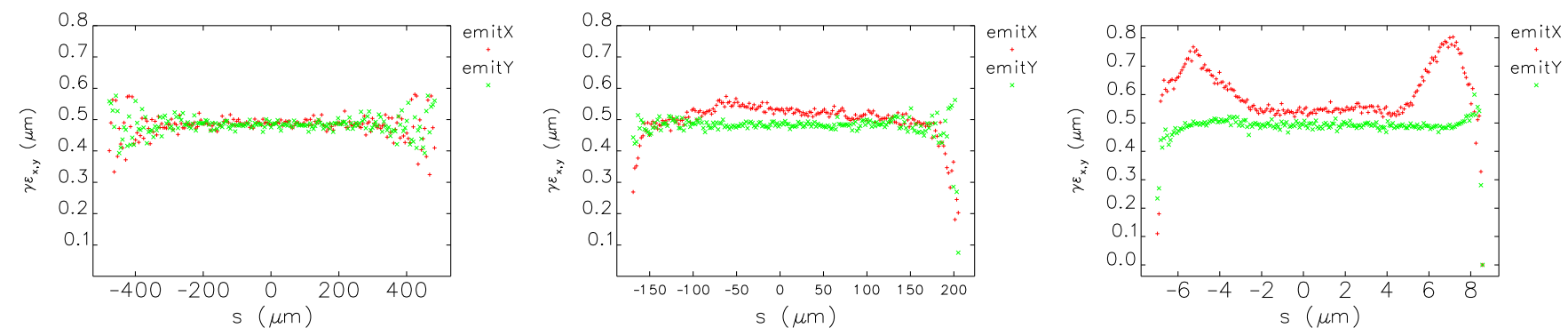

FIG. 6. Longitudinally sliced normalized transverse emittance at different locations. Left: Linac1 end and BC1 entrance; middle: BC1 end and Linac2 entrance; right: BC2 end and Linac3 entrance.

\section{FREE ELECTRON LASER PERFORMANCE}

FEL performance is preliminarily studied, with the GENESIS simulation results presented in this section. A simple model of undulators is adopted without consideration of wake fields and nonlinear optics. In the following parts, we show a concrete example of FEL lasing at $0.15 \mathrm{~nm}$ wavelength, which is the same as achieved in LCLS. The undulator is designed to have a short period of $\lambda_{w}=1.5 \mathrm{~cm}$. The undulator strength $K$ is chosen so that the resonant FEL wavelength is $\lambda_{r}=0.15 \mathrm{~nm}$ at a beam energy of $E=7 \mathrm{GeV}$. The electron bunch distribution generated from ELEGANT simulation is fed into an undulator system, and the associated FEL performance is simulated and evaluated within the code GENESIS [21]. The electron bunch transverse profile is matched into the undulator beam line where the average beta function is roughly 20 meters. No undulator tapering is adopted to increase the FEL saturation power.

Given a high peak current of $3 \mathrm{kA}$, a small sliced transverse emittance and energy spread, the FEL radiation power can saturate within $40 \mathrm{~m}$ long undulator at an average power of $P_{\text {sat }} \sim 10 \mathrm{GW}$, as shown in Fig. 8. In comparison, LCLS achieves $6 \mathrm{GW}$ in 60 meters without tapering. An overall undulator length of $50 \mathrm{~m}$ is chosen to guarantee saturation. The temporal and spectrum profile of the FEL at $40 \mathrm{~m}$ of undulator is shown in Fig. 9 and Fig. 10, respectively. Given the temporal profile of the FEL pulse at $40 \mathrm{~m}$, the total coherent photon number is then

$$
N_{\mathrm{coh}}=\frac{\int P_{\mathrm{sat}}(s) d s / c}{h c / \lambda_{r}} \sim 10^{11} \text { photons/pulse, }
$$



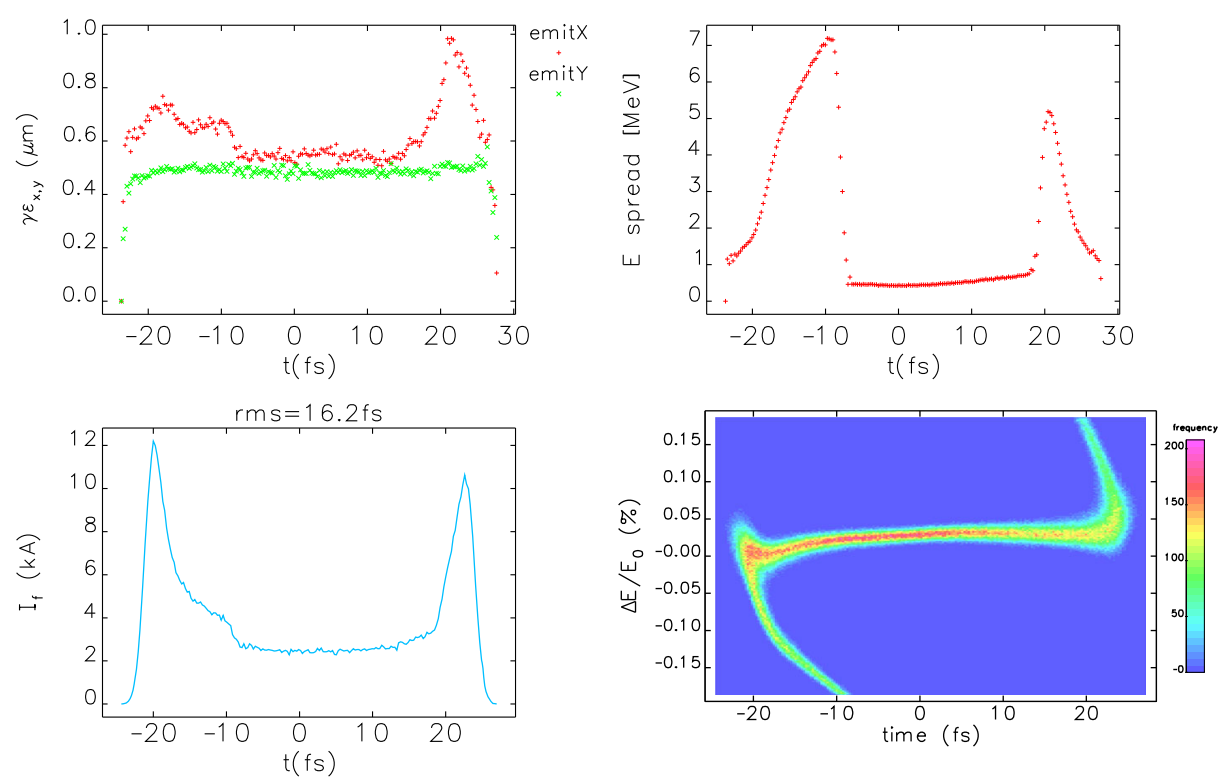

FIG. 7. Beam properties at the end of Linac3 which is also the entrance of the undulator. Left top: longitudinally sliced emittance; left bottom: current profile along longitudinal direction; right top: longitudinally sliced energy spread; right bottom: longitudinal phase space.

where $h \approx 6.626 \times 10^{-34}$, Js is the Planck constant, and $c$ is the vacuum speed of light. As shown in Fig. 9, the FEL temporal profile is a quasiuniform shape, just like the LCLS case. The photon pulse length is roughly $50 \mathrm{fs}$.

As shown in Fig. 10, we fit the FEL power spectrum profile to a Gaussian, and get a bandwidth of $\sigma_{\lambda} / \lambda \sim 4 \times 10^{-4}$. Here the FEL is assumed to be transform limited which gives $\sigma_{\omega} \sigma_{t}=0.5$ for one single coherent mode. If we simply compute the FEL longitudinal phase space area as $\sigma_{\omega} \sigma_{t}$, we have about $\sigma_{\omega} \sigma_{t} \sim 40$, which indicates that there are about $N_{\text {spike }} \sim 80$ coherent temporal spike modes. In comparison, LCLS has roughly 120 coherent temporal spike modes with a slightly longer pulse length of $80 \mathrm{fs}$ and a wider bandwidth. For a SASE FEL, coherent spikes develop during the FEL process along the

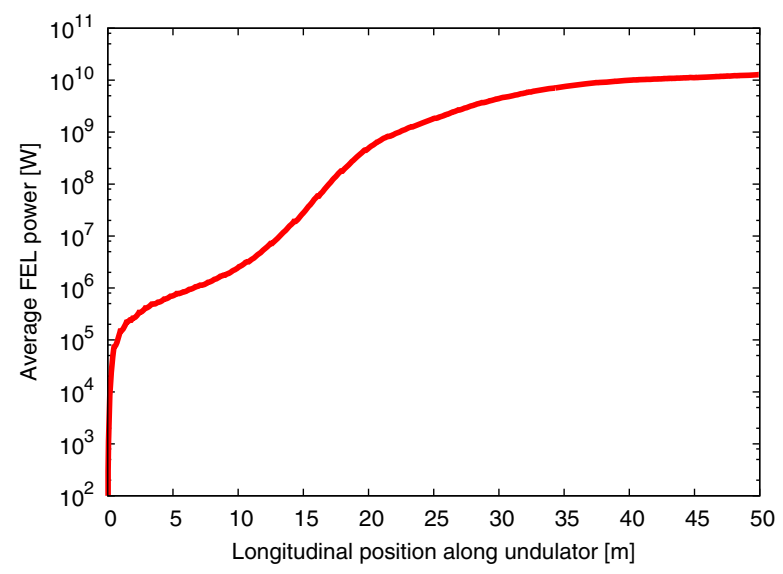

FIG. 8. FEL power evolution along the undulator. undulator. These coherent spikes do not have phase relation, since they start randomly from shot noise.

The FEL radiation wavelength could be tuned in a range of $1.2-0.15 \mathrm{~nm}$ (given same undulator configuration), by changing the electron beam energy between $2.5 \mathrm{GeV}$ and $7 \mathrm{GeV}$. For a soft $\mathrm{x}$-ray configuration with a lower beam energy of $2.5 \mathrm{GeV}$ and a radiation wavelength of $1.2 \mathrm{~nm}$, it should be easier to achieve saturation as the gain length is shorter than the hard $\mathrm{x}$-ray case. The radiation wavelength, the saturation power and length, as well as the wavelength tunable range is comparable to the normal operation mode ( $250 \mathrm{pC}$ bunch charge) of LCLS. The FEL power saturation length of 40 meters is shorter than the LCLS value of 60 meters. The FEL power profile can be tuned by tuning current profile and energy chirp along the bunch (energy profile).

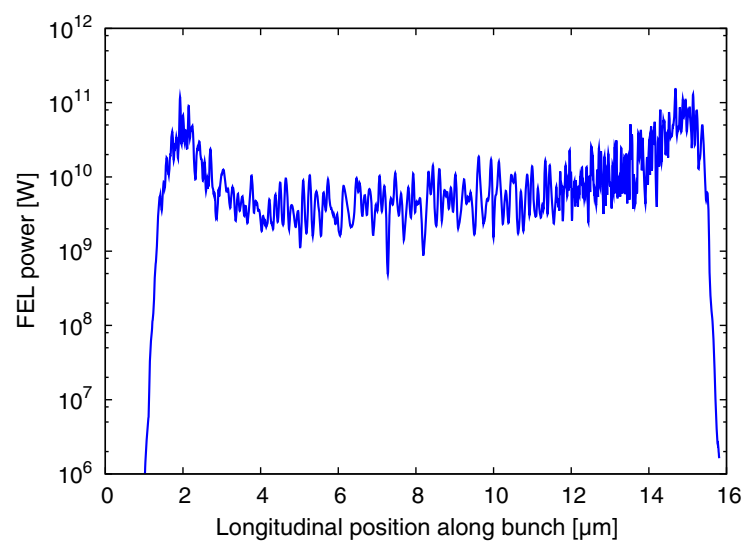

FIG. 9. FEL power temporal profile at undulator $40 \mathrm{~m}$. 


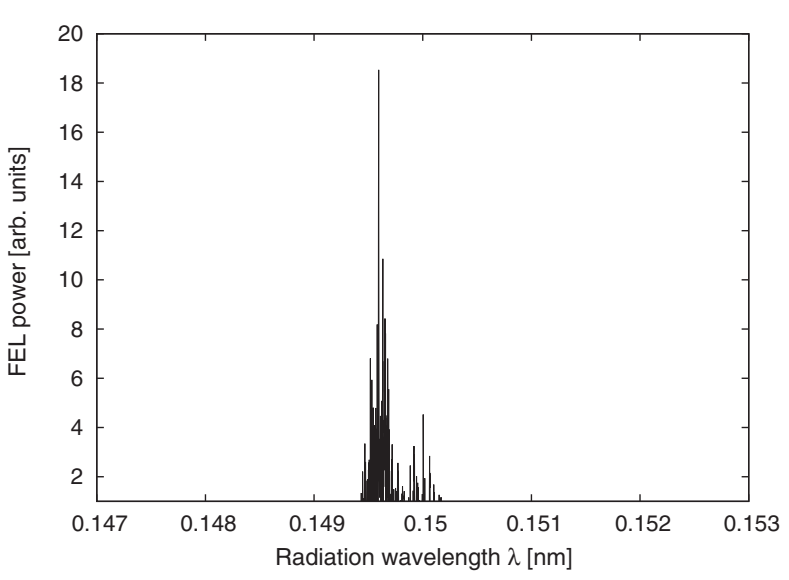

FIG. 10. FEL power spectrum at undulator $40 \mathrm{~m}$.

\section{TOLERANCES}

In this section tolerances on various accelerator components are discussed regarding final beam quality and FEL performance. FEL 1D theory is reviewed to illustrate the relation between FEL performance and electron beam properties. The FEL radiation wavelength depends on the electron beam energy and undulator properties, which can be expressed as $\lambda=\frac{\lambda_{u}}{2 \gamma^{2}}\left(1+\frac{K^{2}}{2}\right)$, where $\lambda_{u}$ denotes the undulator period, $\gamma$ electron beam energy and $K=B[T]$. $\lambda_{u}[\mathrm{~cm}]$ the undulator strength with $B$ the undulator magnetic field. One observes that the electron beam energy needs to be stable to achieve a precise design FEL radiation wavelength. Assuming that similar beam energy feedback loops are adopted for this X-band linac as the LCLS linac, a tolerance of $0.1 \%$ relative final electron energy [7] can be met according to LCLS experiences. The details on beam energy control are not discussed in this paper.

For the self amplified spontaneous emission (SASE) FEL, the radiation power evolvement has three main stages: noisy startup, exponential growth and FEL power saturation stage. In the SASE exponential growth stage, the FEL exponential gain length can be expressed as a function of the FEL parameter $\rho, L_{G} \approx \frac{\lambda_{u}}{4 \pi \sqrt{3} \rho}$. The FEL parameter is related with the beam and undulator parameters and listed below, $\rho \approx \frac{1}{4}\left(\frac{1}{2 \pi^{2}} \frac{I_{p k}}{I_{A}} \frac{\lambda_{u}^{2}}{\beta \epsilon_{N}}\left(\frac{K}{\gamma}\right)^{2}\right)^{1 / 3}$, where $I_{p k}$ denotes electron beam peak current, $\beta$ average beta function in undulator and $\epsilon_{N}$ normalized sliced transverse beam emittance. Usually the SASE FEL power saturates in a length of $L_{\text {sat }} \approx \lambda_{u} / \rho \approx 18 L_{G}$, with a final saturated power of $P_{\mathrm{FEL}, \mathrm{sat}} \approx \rho \times P_{e}$, where $P_{e}$ denotes electron beam power. One observes that given a certain beam power $P_{e}$, the FEL parameter $\rho$ needs to be as large as possible to reach a high SASE saturation power. On the other hand, $\rho$ also needs to be as large as possible to achieve a short gain length $L_{G}$ and then a short saturation length $L_{\text {sat }}$. The impacts on the FEL performance from the electron beam peak current $I_{\mathrm{pk}}$ and the normalized sliced transverse beam emittance $\epsilon_{N}$ are discussed in the following part of this section.
The variation of the electron beam peak current $I_{\mathrm{pk}}$ was studied in great details in [28]. The brief conclusion is that the final peak current variation mainly depends on the bunch charge variation and correlated gun laser to linac rf timing jitter [28]. The achievable bunch charge stability depends on gun laser properties, cathode quantum efficiency and chosen phase with respect to zero-crossing (different between various $\mathrm{X}$-Band guns, such as the 5.59 cell $\mathrm{X}$-Band gun and the 5.5 cell version), which will be measured experimentally on various X-Band guns. Given the LCLS achieved S-Band gun laser stability which provides a charge variation of $1.1 \%$, in the X-Band system a charge variation of $4 \%$ is anticipated in the pessimistic sense. This in turn would increase the FEL power gain length (and saturation length) by $2.5 \%$, and decrease the output FEL saturation power by $6 \%$, estimated under SASE $1 \mathrm{D}$ theory.

It was also summarized that the longitudinal wakefield in Linac2 can be used to partially or fully cancel the timing jitter induced bunch length variation [28], and generate a more constant final electron current profile. This is one possible advantage of X-band rf system over other lower frequency rf systems, as the longitudinal wake field is much stronger in an X-band rf structure. The timing jitter induced peak current variation is largely compensated by decreasing the acceleration gradient in Linac2 and increasing the Linac2 length (employing more rf cavities). The accelerator parameters adopted in this hard X-ray FEL driver are optimum to achieve a shortest accelerator length, which still provide an acceptable FEL performance. Assuming a correlated gun laser to linac rf timing jitter of $25 \mathrm{fs}$ and $50 \mathrm{fs}$, the peak electron beam current and FEL performance variations are calculated by ELEGANT simulation and FEL 1D formulas, as listed in Table I. The tolerances on bunch charge variation and correlated timing jitter are acceptable according to LCLS experience [7].

The normalized transverse beam emittance $\epsilon_{N}$ needs to be as small as possible to achieve a good FEL performance. The accelerator design of this FEL driver is already optimized to preserve the electron beam quality since its generation from the photoinjector. As discussed above, the major source of transverse emittance growth is the CSR effect in the bending systems. Another two possible sources of transverse emittance growth are the quadrupoles and sextupoles misalignment in $\mathrm{BC} 1$, plus linac quadrupoles and $\mathrm{rf}$ structures misalignment. The emittance growth from these misalignments is investigated by ELEGANT

TABLE I. Peak electron beam current and FEL performance variations as a function of correlated timing jitter.

\begin{tabular}{lccc}
\hline \hline Timing jitter & $\Delta I_{\mathrm{pk}}$ & $\Delta L_{G}\left(L_{\mathrm{sat}}\right)$ & $\Delta P_{\mathrm{FEL}, \mathrm{sat}}$ \\
\hline $25 \mathrm{fs}$ & $0.6 \%$ & $0.3 \%$ & $99.7 \%$ \\
$50 \mathrm{fs}$ & $2 \%$ & $1 \%$ & $99 \%$ \\
\hline \hline
\end{tabular}



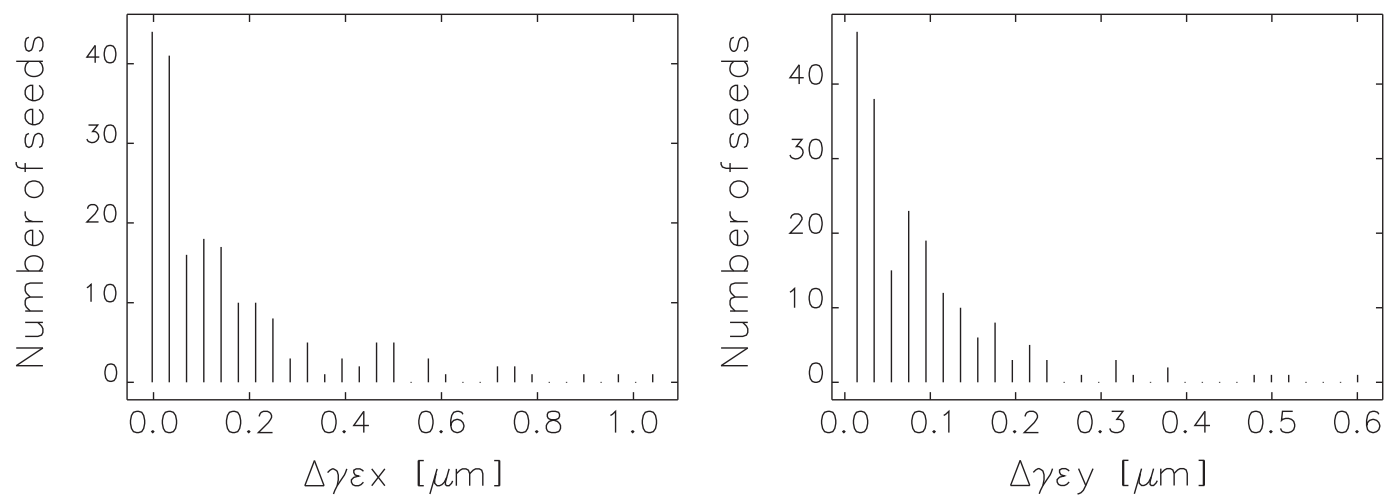

FIG. 11. Left: horizontal projected normalized emittance growth at bunch compressor one end. Right: vertical projected normalized emittance growth at bunch compressor one end. In the simulation $50 \mu \mathrm{m}$ is assumed for the offset between beam position monitor (BPM) electrical center and quadrupole magnetic center. One-to-one steering method (from one quad center to the next) is adopted here.

simulations and illustrated in Fig. 11 and Fig. 12 below. With $200 \mu \mathrm{m}$ RMS offset and $200 \mu \mathrm{rad}$ RMS roll angle errors added on all the quadrupoles and sextupoles in $\mathrm{BC} 1$, a normally compressed electron beam is tracked through Linac1 and BC1 with one-to-one steering. 200 random seeds are employed and an average projected emittance growth of $0.17 \mu \mathrm{m}$ and $0.12 \mu \mathrm{m}$ is found, given relatively weak quadrupoles and sextupoles in $\mathrm{BC} 1$.

The misalignment of the $\mathrm{rf}$ structures and quadrupole magnets in the linac introduce dispersive emittance dilution for a chirped electron beam, plus transverse wake fields induced emittance dilution. Given an optimized linac optics and a short linac length (fewer quadrupoles), the emittance dilution from this effect is not large. Horizontal and vertical offsets with a RMS value of $200 \mu \mathrm{m}$ are generated randomly for all the quadrupoles and rf structures in the linac, while a RMS value of $50 \mu \mathrm{m}$ is assumed for the offset between BPM electrical center and quadrupole magnetic center. An average emittance growth around $0.2 \mu \mathrm{m}$ is found with 200 random seeds for both horizontal and vertical normalized emittance. The longitudinally sliced transverse emittance is weakly affected by the misalignments. The achievement of the required longitudinal

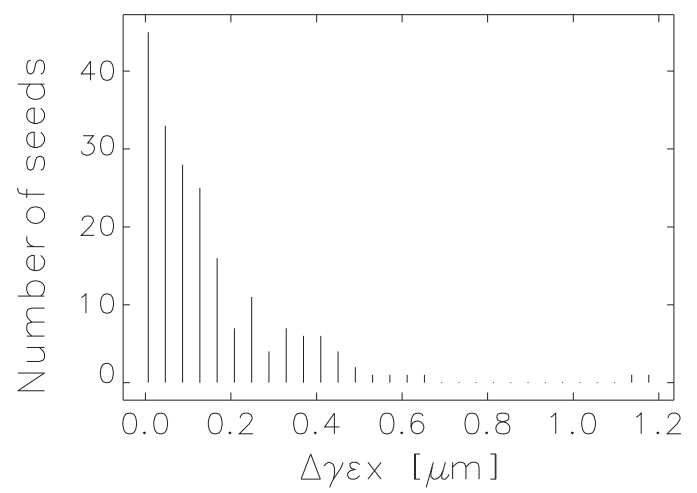

FIG. 12. Horizontal projected normalised emittance growth from misalignment of the rf structures and quadrupole magnets in the linac, observed at linac end. One-to-one steering is adopted. dispersions $\left(R_{56}, T_{566} \ldots\right)$ depends on the magnets (dipoles, quadrupoles, and sextupoles) field quality of the bunch compressors. Additional tuning magnets (normal and skew quadrupoles and sextupoles) could be introduced to correct the longitudinal and transverse dispersions. From the simulations it is observed that the tolerances on the longitudinal dispersions is not tight.

\section{CONCLUSION}

In this paper, an all X-band rf based hard X-ray FEL design is presented with two stage bunch compression. Instead of utilizing harmonic rf for a longitudinal phase space linearization, optics linearization employing specific higher order dispersions is adopted in a first stage bunch compression. The electron bunch is successfully compressed to a peak current above $3 \mathrm{kA}$, while its brightness is well preserved in both transverse and longitudinal plane. The CSR induced transverse emittance growth is on a similar level between this X-band driven FEL and the LCLS. Timing jitter and misalignment could be tolerated in a reasonable manner. FEL simulation indicates that lasing at a wavelength of $0.15 \mathrm{~nm}$ is possible with a short period length undulator. The FEL radiation power saturates in a short length of 40 meters with an average power above $10 \mathrm{GW}$. This all X-band rf based hard X-ray FEL could achieve LCLS-like performance in a shorter overall length of 250 meters.

\section{ACKNOWLEDGEMENT}

Most of the work was done at SLAC National Accelerator Laboratory. The paper was finished at Argonne National Laboratory. Work supported by the U.S. Department of Energy, Office of Science, under Contract No. DE-AC02-06CH11357. Work also supported by the U.S. Department of Energy under Contract DEAC02-76SF00515. The authors would like to thank M. Woodley, A. Chao, Z. Huang, C. Adolphsen, Y. Ding, C. Limborg-Deprey for helpful discussions, C. Adolphsen for the proofreading. 
[1] J. Madey, J. Appl. Phys. 42, 1906 (1971).

[2] D. A. G. Deacon, L. R. Elias, J. M. J. Madey, G. J. Ramian, H. A. Schwettman, and T. I. Smith, Phys. Rev. Lett. 38, 892 (1977).

[3] A. Kondratenko and E. Saldin, Part. Accel. 10, 207 (1980).

[4] R. Bonifacio, C. Pellegrini, and L. M. Narducci, Opt. Commun. 50, 373 (1984).

[5] L.-H. Yu, Phys. Rev. A 44, 5178 (1991); G. Stupakov, Phys. Rev. Lett. 102, 074801 (2009); G. Geloni, V. Kocharyan, and E. Saldin, DESY Report No. 10-033, 2010.

[6] W. Ackermann et al., Nat. Photonics 1, 336 (2007).

[7] P. Emma et al., Linac Coherent Light Source Conceptual Design Report, SLAC-R-593, SLAC, 2002; P. Emma et al., Nat. Photonics 4, 641 (2010).

[8] TESLA Technical Design Report, TESLA FEL 2002-09, DESY, 2002; T. Ishikawa et al., Nat. Photonics 6, 540 (2012).

[9] P. Emma, Report No. SLAC-TN-05-004, 2001.

[10] P. Emma, in Handbook of Accelerator Physics and Engineering 2nd ed., edited by A. W. Chao, K. H. Mess, M. Tigner, and F. Zimmermann (World Scientific, Singapore, 2013), p. 334.

[11] Y. Sun, SLAC Report No. SLAC-PUB-14445, 2011.

[12] T. Raubenheimer, P. Emma, and S. Kheifets, in Proceedings of the 15th Particle Accelerator Conference, PAC-1993, Washington, DC, 1993 (IEEE, New York, 1993).

[13] P. Emma, Report No. SLAC-PUB-1001, DESY Report No. TESLA-95-17, 1995.

[14] P. Emma, Report No. DESY TESLA-98-31, 1998.
[15] W. Decking, G. Hoffstatter, and T. Limberg, Report No. TESLA-2000-40, 2000.

[16] R. England, J. Rosenzweig, G. Andonian, P. Musumeci, G. Travish, and R. Yoder, Phys. Rev. ST Accel. Beams 8, 012801 (2005).

[17] S. Thorin et al., in Proceedings of the 32nd Free Electron Laser Conference, Malmö, Sweden (Max-lab, Sweden, 2010), p. 471.

[18] J. Thompson, Cornell Internal Report No. ERL09-01, 2009.

[19] H. Grote and F. C. Iselin, Report No. CERN/SL/90-13 (AP), 1990.

[20] M. Borland, Advanced Photon Source LS-287 (2000).

[21] S. Reiche, Nucl. Instrum. Methods Phys. Res., Sect. A 429, 243 (1999).

[22] C. Limborg-Deprey et al., in Proceedings of the 24th Particle Accelerator Conference, PAC-2011, New York, 2011 (IEEE, New York, 2011).

[23] Y. Sun, Phys. Rev. ST Accel. Beams 14, 060703 (2011).

[24] D. Carey, The Optics of Charged Particle Beams (Hardwood Academic, New York, 1987), p. 21, p. 123.

[25] K. Brown, Report No. SLAC-R-075, 1982.

[26] K. L. F. Bane, Report No. SLAC-pub-9663, 2003.

[27] Y. Sun, in Proceedings of the North-American Particle Accelerator Conference (NA-PAC13), Pasadena, California, 2013.

[28] Y. Sun, C. Adolphsen, C. Limborg-Deprey, T. Raubenheimer, and J. Wu, Phys. Rev. ST Accel. Beams 15, 030703 (2012). 